\title{
EL SELF CURRICULAR COMO DISPOSITIVO PARA PENSAR LA PERSISTENCIA EN LA EDUCACIÓN DE ADULTOS
}

\author{
THE CURRICULUM-SELF AS A DEVICE TO THINK ABOUT PERSISTENCE IN ADULT EDUCATION \\ O SELF CURRICULAR COMO DISPOSITIVO PARA PENSAR A PERSISTÊNCIA NA EDUCAÇÃP DE \\ ADULTOS
}

\begin{abstract}
Resumen: El artículo presenta los resultados del proyecto "Self curricular en la educación de adultos: reflexiones sobre persistencia en el proceso formativo" cuyo objetivo era reconstruir, mediante la narración oral y escrita, historias de vida escolar de estudiantes adultos en su proceso de formación, destacando las razones para el abandono y/o persistencia en sus procesos formativos. Se trata de una investigación de naturaleza cualitativa mediante un estudio autobiográfico, basada en la obra de William Pinar, estudioso del currículum que desde los años setenta, introduce el tema de la autobiografía para hablar de las experiencias vividas en torno al currículo. Este dispositivo posibilitó la creación de puentes entre lo subjetivo y lo social, favoreciendo una comprensión compartida de las diferentes trayectorias escolares experimentadas por los estudiantes adultos. Fue una estrategia para capturar el currículum narrativo, revelando las diferentes barreras que enfrentan los estudiantes para persistir en sus procesos de formación.
\end{abstract}

Palabras clave: Currículo. Educación de adultos, Self curricular. Persistencia.

Abstract: This article presents the results of the project "Curricular self in adult education: reflections on persistence in the training process", the main objective of which was to reconstruct,

\footnotetext{
${ }^{1}$ Universidade do Estado da Bahia. Salvador, Bahia, Brasil.

${ }^{2}$ University College London, London, United Kingdom.

${ }^{3}$ Universidad de Barcelona. Barcelona, España.
} 
through oral and written narration, the stories of adult education students, highlighting their reasons for abandoning and / or persisting in their studies. The methodology used was qualitative in nature drawing on an autobiographical study, based on Pinar, a scholar of the curriculum who introduced the subject of autobiography to talk about lived experiences of the curriculum. The results reveal that the curricular self is an important device to create bridges between the subjective and the social, favoring a shared understanding of the different school trajectories experienced by adult students. It was a strategy to capture the narrative curriculum, revealing the different barriers faced by students to persist in their training processes.

Key words: Curriculum, Adult education, Self-curricular. Persistence.

Resumo: Este artigo apresenta os resultados do projeto "Self curricular na educação de adultos: reflexões sobre a persistência no processo formativo," cujo objetivo principal era reconstruir, por meio de narração oral e escrita, as histórias da vida escolar de estudantes da educação de adultos, destacando as razões do abandono e / ou persistência em seus processos de formação. Trata-se de uma pesquisa de natureza qualitativa, através de um estudo autobiográfico, baseado em Willian Pinar, estudioso do currículo que, desde a década de 1970, introduziu o tema da autobiografia para falar sobre as experiências vividas em torno do currículo. Os resultados revelam que o self curricular é um dispositivo importante para criar pontes entre o subjetivo e o social, favorecendo um entendimento compartilhado sobre as diferentes trajetórias escolares vividas pelos estudantes adultos. Foi uma estratégia para captar o currículo narrativo, revelando as diferentes barreiras enfrentadas pelos estudantes para persistirem nos seus processos formativos.

Palavras chave: Currículo. Educação de adultos. Self curricular . Persistência.

\section{INTRODUCCIÓN}

La educación de personas adultas (EPA) tiene una acepción amplia que desborda los límites de la escuela y se extiende a casi todos los ámbitos de la vida social. El impacto de la EPA va más allá de la educación formal. Son múltiples los beneficios y despliegues tanto para el individuo como para la comunidad, generando capital humano y apoyando la inclusión social. El individuo integrado en programas de EPA incrementa su participación comunitaria y cívica, mejora su salud física y mental (SCHULLER; p.4. 2017). De esta manera, el fomento de estos programas debe ser un elemento central para la construcción de políticas en diversos departamentos gubernamentales, no sólo en el ámbito educativo (MALLOWS Y DOS SANTOS COSTA, 2019).

Según Haddad y Pierro (2000) la EPA tiene una concepción muy amplia y se desarrolla en diversos espacios de aprendizajes:

Conjunto muy diverso de procesos y prácticas formales e informales relacionadas con la adquisición o ampliación de conocimientos básicos, de competencias 
técnicas y profesionales o de habilidades socioculturales. Muchos de estos procesos se desarrollan de modo más o menos sistemático fuera de ambientes escolares, realizándose en la familia, en los lugares de trabajo, en los espacios de convivencia sociocultural y de ocio, en las instituciones religiosas y, en los días actuales, también a través de los medios de información y comunicación a distancia. (HADDAD Y PIERRO, 2000, p.108, nuestra traducción)

Siendo así, comprender como el adulto percibe su proceso de aprendizaje en distintos espacios educativos tiene un gran valor en su proceso sus formativo porque ayuda a entender las razones por la cuales algunos alumnos abandonan, persisten o permanecen, incluso cuando se encuentran con aspectos que favorecen la exclusión o inclusión en la EPA. Comprender la persistencia del adulto pese a las adversidades tiene un gran valor para entender la educación a lo largo de la vida. La persistencia es entendida aquí como:

La persistencia se extiende a lo largo de una jornada de aprendizaje, a lo largo de la vida, y en toda la vida, e incluye períodos fuera de la educación formal, con una amplia definición de participación que se extiende más allá de la participación en un programa específico de aprendizaje. La persistencia concentra nuestra atención en el aprendiz y en su jornada de aprendizaje, que también puede incluir el autoestudio antes, después, o durante el curso formal del aprendizaje. La persistencia es un proceso de aprendizaje continuo que dura hasta que una persona adulta alcanza sus objetivos educativos. (MALLOWS Y DOS SANTOS COSTA, 2019, p.42)

Por eso, es necesario comprender la trayectoria de los estudiantes adultos, teniendo en cuenta las distintas barreras enfrentadas cuando estos alumnos persisten en sus procesos formativos. Litster (2007) clasifica esas barreras a la participación y a la persistencia en tres grupos:

Cuadro 1 - Barreras a la participación y a la persistencia Litster (2007)

adaptado por Mallows y Dos Santos Costa, 2019

\begin{tabular}{|l|l|}
\hline DISPOSICIONAL & $\begin{array}{l}\text { Actitudes negativas en relación al aprendizaje. Se refieren a las actitudes de } \\
\text { los alumnos en relación al aprendizaje. Estas son frecuentemente informadas } \\
\text { por experiencias y cuestiones anteriores, como poca autoconfianza, falta de } \\
\text { motivación y restricciones emocionales desarrolladas como resultado de } \\
\text { vidas turbulentas e imprevisibles. }\end{array}$ \\
\hline INSTITUCIONAL & $\begin{array}{l}\text { Las barreras institucionales son aquellas que se refieren a las reglas y } \\
\text { procedimientos de la organización en la que el aprendizaje está ocurriendo o } \\
\text { las restricciones relacionadas con el sistema de provisión en sí mismo. Estas } \\
\text { pueden incluir factores como tiempos de clase inconvenientes o intervalos } \\
\text { limitados de provisión. Las cuestiones relacionadas con la acreditación y la } \\
\text { financiación también se incluyen en esta categoría. }\end{array}$ \\
\hline SITUACIONAL & $\begin{array}{l}\text { Las barreras situacionales incluyen los problemas de la vida cotidiana que } \\
\text { impiden la continuidad del estudio, como el desafío de conciliar la vida } \\
\text { familiar, el trabajo y la rutina de la escuela / espacios formativos. Son varias } \\
\text { las barreras: enfermedades, cuidados con niños, problemas familiares, }\end{array}$ \\
\hline
\end{tabular}


problemas de empleo (y preocupaciones muy prácticas, como los costes, el tiempo y la distancia para viajar).

Es necesaria una lectura amplia sobre las distintas barreras a partir de las historias de vida de los sujetos. La persistencia en la educación de adultos debe ser entendida a partir de un análisis integrador que articule las diferentes barreras que impiden que los alumnos sigan el recorrido educativo formal y no formal. No debemos hacer análisis aislados de los diferentes factores que impiden que los alumnos adultos persistan en su educación, responsabilizando a los alumnos o la institución por la permanencia o abandono del itinerario educativo.

Cabe decir que los estudiantes que han permanecido en la EPA ininterrumpidamente constituyen un grupo cuya experiencia escolar no significa haberla vivido de forma tranquila. Al contrario, estos alumnos se han enfrentado a muchos obstáculos durante su experiencia escolar, han superado adversidades tanto fuera de la escuela, como en el interior de la misma, han buscado nuevas rutas y, estratégicamente, han sorteado muchas barreras para salir adelante.

Este artículo tiene como finalidad reflexionar sobre la importancia de las autobiografías en los procesos formativos de la educación de adultos, mediante la presentación del proyecto self curricular como dispositivo importante para crear puentes entre lo subjetivo y lo social, favoreciendo una comprensión compartida de las diferentes trayectorias escolares experimentadas por los estudiantes adultos.

\section{EI SELF CURRICULAR COMO DISPOSITIVO PARA COMPRESIÓN DE ITINERARIOS FORMATIVOS: REFLEXIONES CURRICULARES}

"El currículo es un proyecto de espacios y tiempos subjetivos, con espacios y tiempos sociales, vinculados a los sujetos y sus modos de conversación y un constante interrogatorio crítico de las formas de su construcción" (PACHECO, 2009 p. 398, nuestra traducción). Es un artefacto social que se compone de marcas personales y sociales comprensible en conversaciones complejas y que requiere un análisis de la autobiografía como práctica de investigación.

En la experiencia del currículum se entrecruzan historias del presente, pasado al tiempo que se enfoca hacia el futuro. En el currículum construimos selfs, o sea, conciencia reflexiva. La conciencia del ser o el sí mismo es resultado de multitud de "factores culturales, del momento histórico en que se vive y la sociedad en que se desarrolla"(SEOANE, 2005). 
El self curricular sería un dispositivo para trabajar el autoconcepto, la autoevaluación de la trayectoria educativa, resaltando la travesía entre lo subjetivo y lo social para una mejor comprensión de la constancia en los procesos formativos. Este dispositivo mediador de la comprensión de los suceso y insuceso escolar a partir de una escucha y escritura sistemática y transformadora, por medio de la biografía de sus propias vidas.

Entendemos por dispositivo "aquellos espacios, mecanismos, engranajes o procesos que facilitan, favorecen o pueden ser utilizados para la concreción de un proyecto o la resolución de problemáticas" (SANJURJO, 2009, P. 32). Cuando tomamos el self curricular como dispositivo entendemos que este es un medio relevador de significados, una posibilidad de hacer un recogido en la trayectoria escolar, apuntando puentes entre el subjetivo y el social "un artificio complejo, pensado y/o utilizado para plantear alternativas de acción" (SANJURJO, 2009, p. 32.).

En la educación de personas adultas, trabajar el self curricular implica comprender los factores que favorecen que los alumnos persistan en sus procesos formativos. Ayuda a comprender sus trayectorias extrapolando las variables escolares (dinámicas sociales de desigualdades y exclusión, obstáculos situacionales) y factores pedagógicos y administrativos escolares (gestión del currículo y de la institución formativa, calidad de la enseñanza, financiación, disposición de los espacios formativos). El self curricular nos ayuda a comprender por qué algunos alumnos persisten en su educación siendo ya adultos, a pesar de las adversidades.

\section{EL PROYECTO SELF CURRICULAR: DISEÑO Y DESARROLLO}

El proyecto self educativo tenía por objetivo reconstruir, a través de la narración oral y escrita, las historias de vida escolar, identificando elementos contextuales que propiciaron la interrupción de la trayectoria educativa y las posibilidades para seguir aprendiendo al largo de la vida. Se buscó, por tanto, comprender la trayectoria de los estudiantes adultos, destacando las razones para el abandono y/o continuación de la trayectoria escolar.

El desarrollo del self curricular está basado en un tipo de investigación que traduce el relato de vida escolar. "El relato de vida es una técnica que puede utilizarse de forma creativa, pero que requiere de un encuadre del que emerge su sentido y le otorga rigurosidad. En este sentido, la utilización del enfoque biográfico conlleva numerosas implicaciones metodológicas" (CORNEJO, et al., 2008, p.37). Existen muchas maneras posibles de realizar investigación/formación dentro del 
abordaje biográfico-narrativo. Optamos por trabajar la propuesta de William Pinar orientada de forma reflexiva, a la atención a nuestros narradores y sus trayectorias escolares.

William Pinar es un gran exponente en la reconceptualización del currículo en la década de 1970, en EE.UU. Acuñado por este autor, el método Currere "es el medio para realizar una autobiografía intelectual y personal que busca que el individuo conozca su ser interior, su subjetividad y, bajo ese conocimiento, reconstruya y transforme la realidad social y política" GARCÍA-GARDUÑO, 2014, p.31). Pinar propone una conversación compleja para que los estudiantes se pongan en el centro a sí mismos y pongan también en el centro el mundo que habitan (y al mundo que habita en ellos). Así, el currículo no es método de instrucción centrado en la trasmisión de contenido, sino "la experiencia vivida encarnada, en cuanto, estructurada por el pasado al tiempo que se enfoca en el futuro" (PINAR, 2017, p.56).

Según Pinar( 2017, p.55) el método Currere consta de cuatro fases: regresiva, progresiva, analítica y sintética":

En la fase regresiva uno se vuelve (no se recuerda simplemente) al pasado, o aspectos de él: por ejemplo, la experiencia de la escuela, la experiencia de un maestro o texto influyente, o la relación continuación una disciplina académica. En la fase progresiva uno imagina el futuro (personal, social, político).En la etapa analítica se analizan estos textos y las experiencias que ellos registran y nos proporcionan para entender lo que antes podría haber estado oscurecido por el estar en el presente(PINAR, 2017,p. 55.).

Todas estas fases están interconectadas y son presentadas dentro del enfoque autobiográfico con finalidad de narrar las experiencias vividas en torno al currículo de las personas adultas, articulando aspectos personales, sociales y políticos. Es una estrategia de salir del presente y reflexionar sobre el pasado y las posibilidades de futuro. En síntesis, fase regresiva (pasado), fase progresiva (futuro), fase analítica (comprensión) y fase sintética (movilización).

El método Currere entendido como práctica curricular utilizado en este trabajo, permite comprender la trayectoria curricular, de manera que invita a abrir un espacio para las experiencias subjetivas de formación, reflexividad, contextualización y compromiso de cada estudiante. "Cuando oímos el pasado, nos quedamos sintonizados con el futuro. Entonces, podemos entender el presente que podemos reconstruir, juntos o solos." (PINAR, 2006, p.137).

Por esto, el proyecto self curricular hace referencia a los fenómenos relacionados con la conciencia reflexiva, la conciencia de sí mismo. El propósito es profundizar en el conocimiento de 
los contextos educativos y de los aspectos personales y sociales. Requiere un proceso intencional y de carácter comprensivo. Tiene por finalidad crear conexiones entre los conocimientos, los saberes, las historias vitales y los desarrollos intelectuales y profesionales del estudiante. Se trata de hacer un relato sobre la experiencia formativa vivida en el tiempo y en el lugar teniendo en cuenta las circunstancias personales y sociales.

\subsection{ORGANIZACIÓN DE PROYECTO}

En el marco del proyecto "La Universitat al Barri"4 desarrollado por una escuela de adultos en la provincia de Barcelona, fue encargado un proyecto que conectase los estudiantes de la asignatura didáctica del grado de pedagogía de la Universidad de Barcelona con los estudiantes de la escuela de adultos 5 .

Basado en un enfoque de formación/investigación biográfico-narrativo, "cuando hablamos de investigación biográfica - narrativa, hablamos de un tipo de investigación de tipo cualitativo que se interesa principalmente por las "voces" propias de los sujetos y del modo cómo expresan sus propias vivencias" (RIVAS, 2013, p.81). Por tanto, vinculando las narrativas de los sujetos podemos comprender los contextos en los que se han producido. Como estrategia más adecuada para conectar las distintas y significativas biografías formativas/escolare, presentamos el proyecto "Self curricular en la educación de adultos: reflexiones sobre persistencia en el proceso formativo." El objetivo del proyecto fue reconstruir, a través de la narración oral y escrita, las historias de vida escolar de estudiantes dentro de la educación de personas adultas, destacando las razones para el abandono y/o continuidad en sus procesos formativos y sus expectativas de futuro.

Organizamos la propuesta en cuatro talleres. El primer taller consistía en conocer el grupo y presentar la propuesta del self curricular. En este encuentro presentamos la fundamentación teórica del proyecto y nos aproximamos a los sujetos con preguntas sobre su trayectoria escolar. Discutimos el concepto de self curricular, currículum y educación de adultos. Presentamos los objetivos de la actividad y la necesidad para trabajar autobiografía escolar en la EPA.

\footnotetext{
${ }^{4}$ El proyecto "La Universitat al Barri" (La Universidad en el Barrio) que busca vincular la universidad a experiencias de educación de adultos y educación popular proporcionado intercambios y experiencias de los sujetos de ambas instituciones con la finalidad de construcción de diálogos horizontales.

${ }^{5}$ En este artículo presentaremos solamente el resultado de investigación realizado en la escuela de adultos.
} 
El segundo taller fue práctico, la intención era explicar metodológicamente como se construye la historia de vida. Explicamos el método Currere de William Pinar, destacando las fases y la necesidad de hacer una recopilación curricular y profesional para comprender la identidad de los estudiantes.

Con imágenes, música y poesía hicimos indagaciones sobre la trayectoria escolar. A partir de este debate, reflexionamos sobre la identidad estudiantil de las personas adultas e identificamos barreras enfrentadas en sus procesos formativos, así como los factores que les ayudarán a perseverar en sus caminos.

En el tercer taller, cada estudiante trajo información sobre su vida estudiantil desde un punto de vista positivo a partir de cuestiones propuestas por Puig Rovira (1993, p. 158). "Escribe sobre cómo has sido, cómo eres y como te gustaría ser tu trayectoria escolar; ¿Qué título pondrías a tu biografía curricular y por qué?". Basado en estas preguntas los estudiantes narraran sus trayectorias curriculares y categorizarán de la siguiente forma:

- Mi vida, un pasado con emoción y un futuro con ilusión

- Piedras en los zapatos

- Sueños perdidos / Licenciada en la vida

- Historia de una vida

- El camino se hace al andar

- Mi padre, mi gran maestro

Todas esas autobiografías fueron presentadas en la universidad, en el marco de las actividades de la asignatura Didáctica y Currículum siendo trabajadas con los estudiantes universitarios como ejemplo de autobiografías de personas que, pese a las adversidades, siguen estudiando y poniéndose metas para sus procesos formativos a lo largo de la vida 6 .

Los participantes del taller eran personas adultas de nacionalidad española. Seguidamente presentamos los participantes a través de una breve síntesis sobre aspectos mercantiles y singulares de sus trayectorias educativas.

\footnotetext{
${ }^{6}$ Las estudiantes de la educación de adultos fueron a la universidad presentar sus biografías (self curricular). Después de la presentación y discusión de las narrativas, fue solicitado la construcción de la biografía escolar de los estudiantes de pedagogía. En otro artículo presentaremos las narrativas de los estudiantes de didáctica y currículum sobre su proceso formativo.
} 
Para preservar el anonimato de las participantes, elegimos pseudónimos: cada mujer que colaboró en nuestro estudio es referenciada en el artículo por el nombre de una flor. La intención de este recurso metafórico fue resaltar el carácter poético de la existencia de estas mujeres, de sus narrativas y de sus vidas como un permanente florecer.

Dentro del tema Discusión de los Resultados, profundizaremos en sus narrativas y lo que revelan las interrupciones, el escenario de desigualdades a los cuales se enfrentaron, las experiencias que fueron fundamentales para el retorno a la escuela y como encaran sus proyectos futuros. Seguidamente, presentaremos la caracterización de los sujetos participantes:

\section{VIDA, UN PASADO CON EMOCIÓN Y UN FUTURO CON ILUSIÓN Por Crisálida \\ 60 años, nació en Barcelona y su familia es originaria de Córdoba. Tiene dos hijos. Reflexiona sobre su pasado y futuro desde una mirada positiva. Crisálida demuestra ser una persona resiliente, determinada y dispuesta a reinventarse a través del conocimiento. En su relato señala también cuestiones sociales, de género y del mundo del trabajo. Pasó por la escuela formal de los seis a los catorce años y actualmente participa de cursos de idiomas en la Escuela de adultos y en la Universidad en el Barrio.}

\section{PIEDRAS EN LOS ZAPATOS}

Por Margarita

Nació en un pueblo y en 1972 vino a vivir a Barcelona. Menciona la falta de estímulo y apoyo para estudiar en su juventud y reconoce algunas experiencias en el mundo del trabajo como una gran escuela. En este universo encontró personas que la marcaron y de quienes aprendió no solo conocimientos relacionados a los oficios a los que se dedicó, sino también valores. A pesar de la metáfora en el título de su texto, que sugiere una experiencia con muchos percances, su relato tiene un tono de humor y optimismo. Además, contiene muchas referencias a planes futuros. Volvió a estudiar a los 53 años y resalta haberlo hecho con deseo y alegría.

\section{SUEÑOS PERDIDOS}


Por Rosa

66 años y con dos hijos. Rememora episodios dolorosos de sus primeros años en la escuela, resaltando el carácter opresor de esta institución. Su trayectoria está marcada por muchas adversidades, como la necesidad de asumir diferentes tareas domésticas y trabajar aun cuando era una niña. Rosa solamente frecuentó la escuela formal de los 7 a los 8 años. En su escrito, destaca personas que influyeron en su vida y que le transmitieron la importancia del conocimiento como camino para el empoderamiento personal. Esas vivencias parecen haberla fortalecido para la superación de las dificultades y es probable que le hayan impulsado a volver a la escuela. Ni siquiera el cáncer al que se ha tenido que enfrentar ha frenado su deseo de seguir aprendiendo y de permanecer en la escuela.

\section{PADRE, MI GRAN MAESTRO}

Por Gardenia

68 años, nació en el pueblo de Fuente Vaqueros, provincia de Granada. Allí estudió y vivió hasta los 13 años, cuando se trasladó con su familia a Barcelona. En esta transición tuvo que dejar la escuela. Tuvo dos hijos. El título de su autobiografía destaca la influencia de su padre en su trayectoria de vida y de formación. Volvió a estudiar en 1982 por la necesidad de apoyar a su hija y a partir de ahí ya no paró. Su deseo es seguir aprendiendo y mantenerse en la escuela.

\section{HISTORIA DE UNA VIDA}

Por Jazmín

Jazmín tiene 74 años y resalta los buenos recuerdos de la escuela que frecuentó, una escuela muy progresista, abierta para el mundo, que fomentaba la libertad de expresión a los alumnos. Antes de casarse y de ser madre, trabajó como peluquera en un negocio propio, interrumpiendo temporalmente su vida laboral para cuidar de los hijos. Volvió a trabajar cuando sus hijos crecieron y de forma intermitente buscaba caminos para estudiar y formarse. A pesar de relatar algún mal recuerdo en una escuela de adultos que frecuentó, estos episodios no le quitaron optimismo y ganas de seguir aprendiendo. Después de la jubilación emprendió con su marido lo que considera una gran aventura: viajar. Visitó muchos lugares y conoció culturas diferentes, disfrutando mucho de esta vivencia. Resalta también su pasión por la lectura. Actualmente frecuenta la escuela de adultos y es parte de un grupo de teatro.

EL CAMINO SE HACE AL ANDAR 
Por Lavanda

Con 62 años, Lavanda relata recuerdos muy positivos respecto a sus primeros años en la escuela. Sin embargo, partir de los 6 años, todo cambia y lo que era vivido con alegría, pasó a tener la marca de la opresión: empezó a estudiar en una escuela de monjas: "la educación ahí, volcada solo para chicas, se centraba en la formación de "buenas madres y amas de casa". Estar en un ambiente tan tiránico, donde se estigmatizaba a alumnas entre buenas y malas, ha impactado en su desempeño. Al final, a los 13 años Lavanda dejó los estudios, más por imposición de su familia y por sugerencia de la propia escuela, que por deseo proprio. A pesar de intentar volver a la escuela a la muerte de su padre, se vio obligada a asumir el sustento de la familia, con tan solo 15 años. Lo que parecía ser un punto final a su deseo de seguir aprendiendo se convirtió en una oportunidad: el mundo del trabajo le propició experiencias formativas muy relevantes, incluso en espacios formales de educación. Lavanda relata también los dilemas vividos por la mujer para conciliar matrimonio, maternidad, trabajo y estudios. A pesar de haber interrumpido su camino formativo muchas veces, subraya su capacidad de reinventarse.

\section{DISCUSIÓN DE LOS RESULTADOS}

El self curricular fue un dispositivo de aprendizajes sobre el recorrido de las personas adultas en sus trayectorias escolares. Por medio de esta estrategia, los participantes en el proyecto se dieron cuenta de las múltiples razones para el abandono y/o persistencia en sus procesos formativos. Los resultados señalizan que las narrativas escolares sobre las razones del abandono de la escuela influenciaron en el empoderamiento y fortalecieran el deseo de seguir estudiando.

El análisis de los relatos autobiográficos seguirá los siguientes pasos: a) Lectura del material; b) Segmentación en Unidades Temático; c) Identificación de las principales dimensiones y categorías; d) análisis de las historias.

Las categorías que hemos encontrado para comprender las razones para el abandono y/o continuidad en sus procesos formativos fueron: otros caminos (el abandono escolar) y nuevos caminos (la persistencia en los procesos formativos).

\section{a) Otros caminos... (el abandono escolar)}


Las narrativas que presentamos a continuación explicitan vivencias y experiencias que han sido decisivas en la interrupción de la trayectoria escolar de las participantes del estudio, resaltando las diferentes barreras enfrentados por esas mujeres a lo largo de su trayectoria escolar.

Tenía 15 años y estaba a punto de cumplir los 16, un hecho que marcó mi vida, mi padre murió de un infarto y me volví en la pieza principal del sustento de la familia, yo era la mayor y mi hermano tenía 7 años. Unos vecinos muy amigos me ayudaron a entrar a trabajar en un laboratorio farmacéutico que él trabajaba, primero estuve en planta fabrica y me fueron preparando para hacer faenas más técnicas, al año me pasaron al laboratorio de control y Análisis, allí se me abrió un mundo nuevo, me enseñaron a analizar, primero cosas sencillas, luego cosas más técnicas.... Tenía 17 años cuando volví a estudiar y me sentía muy bien y valorada... El destino me tenía preparado otro camino, en el segundo curso conocí al que sería mi marido, era un estudiante de último curso de maestría de otra rama, cuando terminé mi oficialía nos casamos y tomé la decisión de parar de estudiar de momento, que nunca pude retomar, me casé a los 21 años y tuve a mi primer hijo a los 22 y mi segundo a los 24 años, cuando tenía 25 años decidí dejar de trabajar, me sentía presionada por mi entorno y lo que yo quería (cuidar de mis hijos ), mi trabajo me gustaba mucho, pero lo dejé; a los 28 años nació mi tercera hija. (Lavanda)

La narrativa transcrita de Lavanda resalta como las barreras situacionales fueron decisivas para interrumpir su proceso de escolarización. Inicialmente, como consecuencia de la muerte de su padre, contingencia esta que le obligó a asumir el rol de mantener económicamente la familia e ingresar en el mundo laboral. Más tarde, a pesar de haber tenido la oportunidad de retomar los estudios, se encontró con nuevos dilemas para conciliar la vida familiar, los estudios y el trabajo. Cabe destacar que, a través de su relato, percibimos como el trabajo se convirtió para ella en un espacio de aprendizaje y formación fundamental.

Como Lavanda, Crisálida, Rosa y Gardenia también se enfrentaron barreras situacionales que repercutieron en sus trayectorias formativas.

Tuve que dejar el colegio y ponerme a trabajar para poder ayudar a mi familia. Con los 14 años recién cumplidos, entré a trabajar en una fábrica de ropa como aprendiza; el horario era de 6,30 de la mañana a $15 \mathrm{~h}$ de la tarde. Como era menor de edad, mi padre tuvo que firmar el contrato de trabajo. Una aprendiza era una persona que se pasaba todo el día de arriba y abajo haciendo recados, por un sueldo de 500 ptas. y 25 ptas. la hora extra; con el tiempo todo eso me valió para aprender a manejar diferentes máquinas y a coser bien. Al final me gustaba mucho. Estuve trabajando casi 40 años en la misma empresa, hasta que el local cerró (Crisálida)

Durante muchos años, puesto que no había trabajo en España y muchas personas emigraron a Catalunya como forma de sobrevivir, era muy duro y por eso yo fui solamente un año al colegio, en la edad de 7 a 8 años. Tenía que cuidar de mi madre que estaba enferma y después nació mi hermana y mi madre no trabajaba. Con 11 años empecé a trabajar, aunque no era legal. No se podía comenzar hasta los 14 
años. Como era muy espabilada siempre buscaba un mejor sueldo y no me importaba cambiar a menudo de trabajo. $Y$ entonces mi padre que era carpintero, en las maderas de la carpintería me enseñó a multiplicar y dividir como el mejor maestro, y mientras trabajaba me ayudaba si tenía alguna duda. Esto lo recuerdo con mucho cariño. (Rosa)

A los 13 años nos vinimos a Barcelona empecé a trabajar. Dejé el colegio. Se necesitaba mi sueldo. (Gardenia)

Estos relatos biográficos se conectan a aspectos socio-contextuales. Una de las cuestiones, por ejemplo, se refiere a la dimensión de género, lo que apunta a desigualdades en la distribución de tareas domésticas y familiares entre hombres y mujeres. La posguerra y el consecuente contexto de precariedad en la que España se sumergió, también fueron mencionados como aspectos que marcaron la trayectoria de estas mujeres.

\section{b) Nuevos caminos (la persistencia en los procesos formativos)}

En los siguientes relatos, observamos el valor que los participantes atribuyen al retorno de las trayectorias interrumpidas, es decir, sus trayectorias de estudios:

He decidido retomar cosas que dejé pendientes, así que he vuelto a la escuela de adultos, con 58 años, para seguir estudiando. Estoy aprendiendo catalán, antes estaba prohibido. Ahora tengo dos nietos que hablan catalán y me lo he propuesto para hablar con ellos. También estoy aprendiendo inglés, por si tengo la oportunidad de viajar en un futuro...Por eso yo pienso que las persones no prestamos mucha atención cuando nos enseñan, luego con los años empiezas a disfrutarlo y en el futuro esperas seguir aprendiendo mucho, durante mucho tiempo (Crisálida)

Con 53 años, volver a hacer deberes, estudiar y encima con alegría no por obligación. Días en que despertaba a las 3 pm y ponerme delante del ordenador por que tenía que sacar el proyecto y no podía dejar de pensar. (Margarita)

Estas voces reflejan que el aprendizaje es un proceso continuo y se realiza en múltiples espacios. Hay un deseo que moviliza al sujeto a afrontar las adversidades, sean institucionales o situacionales. Los sujetos disfrutan con el aprendizaje, se sienten reconocidos y fortalecidos para seguir adelante.

Los relatos de vida señalan que el retorno a los estudios involucra dos dimensiones: una de ellas se refiere al ámbito de la curiosidad y a la búsqueda del saber y el conocimiento. Las participantes cuentan cómo y porqué fueron al encuentro de los espacios formativos, destacando el interés para explorar y experimentar diferentes ámbitos de saberes: cultura y arte, idiomas, informática, ortografía, matemáticas... Por otro lado, matizan estos entornos formativos como locus 
privilegiados para reinventase, ampliar horizontes y perspectivas sobre la vida, construir y fortalecer vínculos de amistades.

Empecé otra vez a estudiar en una escuela de adultos, tenía que aprender catalán para ayudar a mi hija en los deberes en catalán. Me animé y me puse a estudiar de nuevo. Me siento orgullosa del premio que gané para Sant Jordi de Narrativa. Me saqué el graduado escolar que no lo tenía, trabajaba, llevaba mi casa y saqué tiempo para estudiar. Empecé a trabajar en un centro médico de limpieza y mantenimiento. Después de llevar 22 años en la misma empresa trabajando cerraron dos centros donde yo trabajaba. Me propusieron el despido o que me formara para ganar un ordenador. Opté por aprender informática. Vine a la escuela de adultos CFA Canyelles y me saqué el título de informática Competic3 estudiando dos días por semana. Seguí trabajando. Hice soporte administrativo, chófer de los coches del SEM y su mantenimiento. Me jubilé. Sigo en la escuela haciendo Universitat al Barri. No puedo hacer más cosas por tener que ir a cuidar a mis nietos tres días a la semana a Calella de Mar. Mi idea es seguir aprendiendo y no dejar el colegio. Tenemos un gran grupo de amigas, de lo mejor, compañeras para todo (Gardenia)

Soy una persona que siempre me ha gustado leer y tener un libro en mis manos. He sentido curiosidad por muchas cosas y he intentado informarme. Durante muchos años me sentía mal por no haber seguido estudiando y con mis inseguridades no me valoraba. Fomenté a mis hijos el estudio y la cultura y me siento muy orgullosa que los tres tienen estudios universitarios. Con la madurez y haciendo un recorrido por mi vida me doy cuenta que el valor en mi es que me he sabido reinventar, he tomado el interés de aprender sobre el tema que he trabajado para poder hacerlo mejor, el trabajo puede ser una buena escuela si se toma interés de aprender, nunca paramos de aprender. He vuelto a escuela, he encontrado en el barrio una escuela de adultos, estoy muy contenta, hay muy buenos profesores y participó en clases sobre cultura, historia, memoria y cálculo mental, ortografía castellana y catalán, mi asignatura pendiente, en mi juventud estaba prohibido y no lo estudie, ahora es un buen momento; participo en las clases de la Universidat al Barri, son muy interesantes y amenas. He empezado a tomar clases de pintura al óleo, me encanta y me sorprende mi evolución. (Lavanda)

Cuando dejé de trabajar pensé que era el momento de volver a la escuela. Me dijeron que tenía una cerca de casa y allí me matricule en informática con mucha alegría pues me gustaba mucho. Así que me puse manos a la obra y cuando llevaba dos años un cáncer intentó frenarme, pero por poco tiempo. Mis compañeras hicieron piña y no me dejaron hundirme y así cuando me daban la quimio faltaba dos días y al tercero con mi pañuelo ya tenía que ir a las clases. Mis compañeras y los profesores me daban vida, fue muy bonito. Me ayudaron cuando me perdía en los trabajos que al final pude sacar adelante. Ahora mi inquietud es seguir aprendiendo de lo que la vida me trae. Mis ilusiones siguen intactas, me gusta ver el cielo por la noche tanto si hay luna como si no, las estrellas y los planetas, estar en mi balcón y disfrutar de mis plantas. (Rosa) 
Así, las historias de vida de estas mujeres retratan que el proceso de aprendizaje no es lineal y tampoco está relacionado solamente con los tiempos y espacios de la educación formal. Se trata de un proceso que, por así decirlo, florece a lo largo de toda la vida.

Creo que el saber te abre la mente y te das cuenta que hay mucho por aprender. Me gustaría seguir teniendo y no perder las ganas por aprender y encontrar nuevos conocimientos. No estoy interesada en obtener títulos, sólo conocimientos. (Lavanda)

\section{ULTIMAS PALABRAS}

Los resultados revelan que la travesía entre lo subjetivo y lo social, por lograr una comprensión compartida de estudiantes en la educación de adultos, es una tarea fundamental para entender la persistencia en los procesos formativos y para crear estrategias didácticas que puedan asegurar la educación a lo largo de la vida.

El self curricular es un dispositivo para expresar emociones a través de la palabra escrita y oral; es un medio a partir del cual las identidades individuales y sociales pueden ser representadas, fortalece la autoestima, fomenta el protagonismo de las personas, posibilita un reencuentro con el pasado y abre ventanas para el futuro. Fue una estrategia a la hora de captar el currículum narrativo:

... para captar así el currículum vivo, narrativo, que se va entretejiendo en la vida de una clase, y para poder pensar tanto sus tensiones y dificultades, como sus posibilidades para una enseñanza que se tenga en cuenta lo que va sucediendo. Hablar de un currículum narrativo supone poner el acento, no en los documentos prescriptivos ni en su aplicación, sino en las experiencias que se viven en las aulas. El currículum es narrativo en la medida en que la vida del aula se compone de situaciones que van evolucionando. (CONTRERAS, et al 2019, p.33)

Así fue la historia de los estudiantes adultos. Todas las participantes se han encontrado y aún se encuentran con innumerables barreras situacionales que continúan dentro de sus circunstancias vitales. Desigualdades sociales y culturales como por ejemplo el desafío de conciliar la vida familiar, el trabajo y la rutina de la escuela / espacios formativos son algunas de ellas. Sin embargo, todas han seguido cultivando el deseo por volver a la escuela y han podido construir un camino para este retorno.

- Las experiencias negativas en la educación primaria y/o secundaria no han destruido el deseo por retornar y por vivir nuevas experiencias formativas. 
- La escuela de adultos adaptada al tiempo de las participantes, facilita una mayor implicación en el proceso formativo porque tiene en cuenta las dificultades a las que estos sujetos se enfrentan, especialmente las barreras institucionales y situacionales.

- Resaltamos que los cursos ofertados por las escuelas de jóvenes y adultos fueron concebidos para atender los deseos y necesidades de este público y al mismo tiempo para propiciar un espacio de encuentro y fortalecimiento de vínculos.

En la educación de personas adultas, trabajar el self curricular implica comprender los factores que favorecen que los alumnos persistan en sus procesos formativos. Ayuda a comprender sus trayectorias extrapolando las variables escolares (dinámicas sociales de desigualdades y exclusión, obstáculos situacionales) y factores pedagógicos y administrativos escolares (gestión del currículo y de la institución formativa, calidad de la enseñanza, financiación y disposición de los espacios formativos). El self curricular nos ayuda a comprender por qué algunos alumnos insisten en su educación como adultos, a pesar de las adversidades.

Con el self curricular se evidenció que la disponibilidad para seguir estudiando fue fundamental en el proceso formativo. Como nos comenta una estudiante: "En el futuro espero poder seguir aprendiendo y disfrutando de todo lo que la vida me vaya aportando" (Crisálida).

\section{REFERENCIAS}

CORNEJO, M., ROJAS, R.C. \& MENDOZA, F. La investigación con Relatos de Vida: Pistas y opciones del Diseño Metodológico. Psykhe,2008. 17, 29-39.

CONTRERAS D, J.; QUILES-FERNÁNDEZ, E.; PAREDES SANTÍN, A. Una pedagogía narrativa para la formación del profesorado. Márgenes, p. 58-75, 29 jul. 2019.

HADDAD, Sérgio; DI PIERRO, Maria Clara. Escolarização de jovens e adultos. Revista Brasileira de Educação, São Paulo, Anped, n. 14, p. 108-130, mai.-ago.2000.

GARCÍA-GARDUÑO, J. M. Estudio Introductorio, en W.F. Pinar, La teoría del currículum. Narcea: Madrid, España.2014.

MALLOWS, D; DOS SANTOS COSTA, G. Persistencia en la educación de jóvenes y adultos: reflexiones sobre currículo e inclusión. In Educación e inclusión: desafíos formativos y curriculares. Barcelona: Ediciones Zaragoza, España,2019. 39-53.

PINAR, W. Currere: aquel primer año. Investigación Cualitativa, 2(1) pp. 55-65, 2017. DOI: http://dx.doi.org/10.23935/2016/01035. Disponible en: 
https://ojs.revistainvestigacioncualitativa.com/index.php/ric/article/view/59. Accesso en set de 2019

PINAR, W. F. A política de raça e gênero da reforma curricular contemporânea nos Estados Unidos. Currículo sem Fronteiras, v.6, n.2, p. 126-139, Jul/Dez 2006. Disponible en: www.curriculosemfronteiras.org. Acceso en: 04 abr. 2019.

PACHECO, J. A. Currículo: entre teorias e métodos. Cadernos de Pesquisa, São Paulo, v.39, n.137, p.383-400, 2009. Disponible en: <http://dx.doi.org/10.1590/S0100-15742009000200004>. Acceso en: 19 nov. 2019.

SEOANE, J. Hacia una biografía del self. Boletín de Psicología, 85, 41-88.2005

SANJURJO, L. Los dispositivos para la formación en las prácticas profesionales. Rosario, Argentina: Homo Sapiens Ediciones.2009.

SCHULLER, T. What are the wider benefits of learning across the life course? Government Office for Science: London.2017

PUIG ROVIRA, J. Ma. Notas para un estudio sobre los usos de la escritura autobiográfica en educación. Pad'e, vol. 3, ns 1, pp. 153-162.1993.

RIVAS, J. I. La investigación biográfica y narrativa.2013Disponible en http://www.juntadeandalucia.es/averroes/impe/web/contenido?pag=/contenidos/B/Innovacion Elnvestigacion/InvestigacionEducativa/Materiales/nvestigacionEducativa/Seccion/InvestigarEnEdu cacion/T207Biografias. . Acceso en el 11 de octubre 2019.

RIVAS, J. I. Narración, conocimiento y realidad. Un cambio de argumento en la investigación. En, Voz y educación. La narrativa como enfoque de interpretación de la realidad. Barcelona: Ediciones Octaedro, S.L.2009.

\section{SOBRE LOS AUTORES:}

\section{Graça dos Santos Costa}

Doctora en Pedagogía por la Universidad de Barcelona (UB). Profesora titular de la Universidade do Estado da Bahia (UNEB) y profesora asociada de la Universidad de Barcelona (UB).

E-mail: gracacosta@gmail.com

Orcid: https://orcid.org/0000-0001-7770-0118

\section{David Mallows}

Doctor of Philosophy of Teacher Education, Principal Teaching Fellow, UCL Institute of Education. E-mail: d.mallows@ucl.ac.uk

Orcid: https://orcid.org/0000-0001-8378-5488

\section{Juliana Machado De Carvalho}

Doctoranda del Dpto. de Didáctica y Organización Educativa de la Universidad de Barcelona (UB). 
Master en Comunicación y Educación por la Universidad Autónoma de Barcelona (UAB).

E-mail: julianamsans@gmail.com

ORCID: https://orcid.org/0000-0001-8111-8884

Recebido em: 29 de maio de 2020

Aprovado em: 22 de junho de 2020

Publicado em: 01 de julho de 2020 